\title{
Dollfustravassosius moniliovatus gen. n., sp. n. (Trematoda, Isoparorcbiidae) un Trématode parasite de Poisson marin
}

\author{
Par J. F. TEIXEIRA DE FREITAS et Anna KOHN
}

Au mois de mars de 1963, les Professeurs L. Travassos, A. Kohn et C. da Silva Motta, avec l'auxiliaire de la Section d'Helminthologie de la Division de Zoologie de l'Institut Oswaldo Cruz, M. José Walter dos Santos, ont séjourné pendant une semaine à l'Ecole technique de Pêche Darcy Vargas. Cette Ecole est située dans l'île de Marambaia, dans la région sud du littoral de l'Etat de Rio de Janeiro, au Brésil.

Pendant les recherches helminthologiques effectuées, furent collectés cinq exemplaires d'une espèce de Trématode dans la vessie natatoire d'un poisson marin, connu par la population locale sous le nom de « bagre urutu ». Ces spécimens sont enregistrés par Travassos et ses collaborateurs (1963) dans la note sur cette excursion, publiée dans les comptes rendus (Atas) de la Société de Biologie de Rio de Janeiro, sous la dénomination générale de Trématodes (page $6,1^{\text {ro }}$ colonne, lignes 2-4: in Tachysuridae; lignes 6-8: in Tachysurus grandicassis). Les $T$. grandicassis autopsiés furent enregistrés sous les numéros: 18.311, négatif ; 18.343, négatif ; 18.390 , avec trois exemplaires mutilés ; 18.410 , avec un exemplaire complet, et 18.425, avec un exemplaire intégral.

Ces cinq exemplaires (deux complets, l'un sans la partie postérieure du corps et les deux autres mutilés par des ciseaux pendant la nécropsie) furent récemment étudiés par nous, après avoir été colorés par le carmin et diaphanisés par le créosote de hêtre ; ils représentent une espèce nouvelle pour la science, laquelle doit être classée dans un nouveau groupement générique d'une nouvelle sous-famille, proche de la sous-famille Isoparorchiinae (Travassos, 1922) (1).

(1) Dollfus, en 1959, dans un travail sur l'agent pattogène de la maladie de la «tache d'encre » chez des poissons du Vietnam, a présenté un bel aperçu historique de l'espèce Isoparorchis hypselobagri (Billet, 1898) Ejsmont, 1932. Dans ce travail on voit qu'il y a, dans la littérature, quelque confusion sur la date de la sous-famille Isoparorchiinae, proposée par Travassos.

Nous pouvons, en ce moment, expliquer les faits passés et débrouiller la confusion qui existe sur cette date :

Pendant la session du 13 décembre 1920 de la Société Brésilienne des Sciences (Section de Sciences Biologiques), Travassos a présenté un mémoire sur les Trématodes de la famille des Gorgoderidae, comprenant l'historique, la systématique, l'anatomie et la distribution de ces Helminthes. Le compte rendu de cette session a été publié, anonymement, dans la \& Folha Medica (année 1, 


\section{DOLLFUSTRAVASSOSIINAE subfam. n.}

\section{Isoparorchiidae.}

Trématodes grands ; ventouse orale, pharynx et acétabulum bien développés ; caeca intestinaux sinueux; pore génital œsophagien; atrium génital (ducte hermaphrodite) présent ; poche du cirre absente ; prostate courte; vésicule séminale développée; testicules allongés, post-acétabulaires et latéraux; ovaire intercaecal, post-testiculaire ; utérus pré-ovarien; œufs operculés, accolés en filaments moniliformes; vitellogènes en groupes de follicules en grappe, au niveau de la région de l'ovaire; vessie excrétrice en $\mathrm{Y}$, avec des branches anastomosées antérieurement. Parasites des poissons marins.

GENRE TYPE: DOLLFUSTRAVASSOSIUS gen. n.

\section{DOLLFUSTRAVASSOSIUS gen. n.}

\section{Dollfustravassosiinae.}

Corps avec l'aspect fascioliforme. Cuticule fine et lisse. Ventouse orale sous-terminale. Acétabulum pré-équatorial. Pharynx présent. Esophage court. Caeca intestinaux sinueux. Pore génital ventral à l'œsophage. Atrium génital (ducte hermaphrodite) présent, allongé. Poche du cirre absente. Cirre petit. Région prostatique courte. Vésicule séminale bien développée, sinueuse, pré-acétabulaire. Testicules allongés, post-acétabulaires, latéraux, pré-ovariens, dans les aires extracaecales, caecales, et intercaecale. Ovaire plus ou moins arrondi, rapproché de l'extrémité postérieure du corps, intercaecale. Glande de Mehlis présente, près de l'ovaire, Réceptacle séminal féminin (spermathèque) et canal de Laurer non observés. Utérus pré-ovarien, intercaecal, caecal et extracaecal. Eufs operculés, petits, accolés en filaments moniliformes. Vitellogènes constitués par groupes de follicules en grappe, intercaecaux, caecaux et extracaecaux ou, trépassant antérieurement et postérieurement la zone de l'ovaire, pore excréteur terminal. Vessie excrétrice en forme d'Y, avec des branches sinueuses qui s'anastomosent au niveau du pharynx. Parasites des poissons marins.

ESPÈCE TYPE: D. moniliovatus sp. n.

$\mathrm{n}^{\circ}$ 21-24, 16 décembre 1920, pp. 188-189); on n'y trouve aucune information sur les groupements systématiques proposés par Travassos.

Dans le $\mathrm{n}^{\circ} 2$ du volume 1 de l'année 36 du «Brasil Medico», publié en janvier 1922 (pp. 17-20) a paru un travail de Travassos intitulé : «-Contribuições para o conhecimento da fauna helmintológica brasileira. Espécies brasileiras da família Gorgoderidae Looss, 1901 \%. On y voit, pour la première fois, l'établissement de la nouvelle sous-famille lsoparorchinae (sic), qui, à la page 19 est mentionnée avec une erreur typographique : Isoparorchinas.

Au mois de décembre de 1922 , est publié le fascicule 1 du tome XV des Mémoires de l'Institut Oswaldo Cruz, où l'on trouve un autre travail de Travassos: \&Contribuições para o conhecimento da fauna helmintológica brasileira. XVII. Gorgoderidae brasileiras (Contributions à l'étude de faune helminthologique du Brésil. XVII. Gorgoderidae brésiliennes) », dans les pages 220-234 (pp. 125137 ) et avec les planches 26-30, figures 1-32. Dans ce travail Travassos informe qu'il fut présenté à la Société Brésilienne des Sciences en juin de 1920 (ce mois est un lapsus) et retire la sous-famille Isoparorchinae de la famille Gorgoderidae; cette sous-famille est considérée, alors, dans les Hemiuridae (cf. pp. 221, $230 ; 126,135-136$ ). Travassos a cité comme l'auteur de la sous-famille: Travassos, 1920 , ce qui n'est pas exact.

Pendant le mois de janvier 1923, la typographie de l'Institut Oswaldo Cruz a composé les couvertures de cette publication, comprenant les textes en portugais et en français; dans ces couvertures fut imprimée la date de 1923 et aussi : «Réimpresso das (Réimpression des) Memórias do Instituto Oswaldo Cruz, tomo XV, fac. I, 1922) s.

D'après cet exposé nous pouvons conclure : 1) qu'il n'y a aucune publication de Travassos sur cette sous-famille pendant l'année de $1920 ; 2$ ) que la sous-famille des Isoparorchiinae fut établie en janvier 1922, sous la graphie Isoparorchinae; 3) qu'il n'y a pas de publication sur cette sousfamille faite par Travassos en 1923. 


\section{DOLLFUSTRAVASSOSIUS MONILIOVATUS sp. n.}

Trématodes d'aspect fascioliforme, avec une partie antérieure étroite et une partie postérieure plus large et plus longue, atténuée à son extrémité ; la cuticule est fine et lisse. Taille : 19,66 à 32,66 mm de long sur 7,06 à 10,47 mm de large. La ventouse orale est sous-terminale, dépassée antérieurement par la paroi du corps; elle a de 0,70

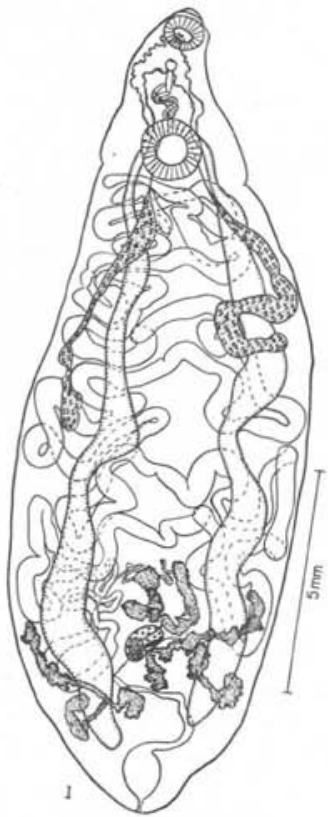

FIG. 1. - Dollfustravassosius moniliovatus gen. n., sp. n. : L'exemplaire type

FIG. 2. - Dollfustravassosius moniliovatus gen. n., sp. n.: Paratype $\mathrm{n}^{\circ} 30.081$

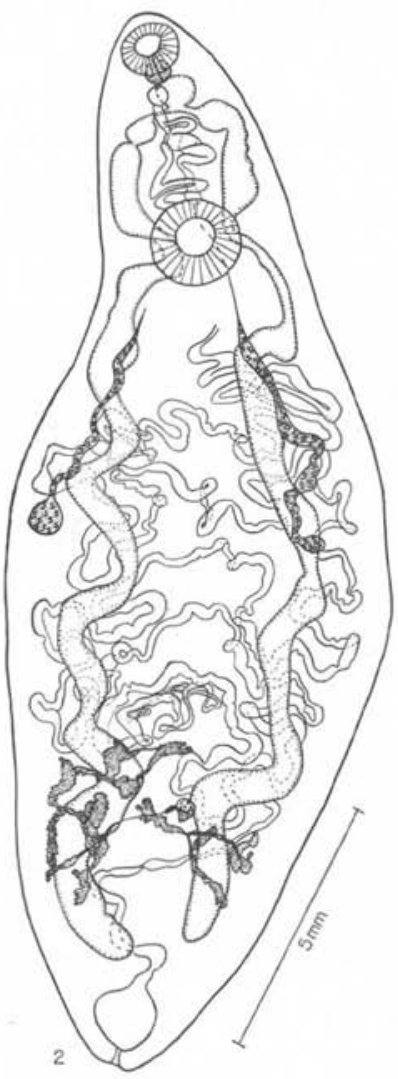

à $1,33 \mathrm{~mm}$ de diamètre longitudinal sur 0,74 à $1,53 \mathrm{~mm}$ de diamètre transversal. La ventouse ventrale ou acetabulum est située dans la partie antérieure du corps; son diamètre longitudinal est de 0,96 à $2,47 \mathrm{~mm}$ et son diamètre transversal de 0,94 à $2,47 \mathrm{~mm}$. Le rapport entre la ventouse orale et l'acetabulum est variable de $1: 1,53$ à $1: 1,72$. Il n'y a pas de prépharynx. Le pharynx est musculeux, long de 0,32 à $0,60 \mathrm{~mm}$ et large de 0,36 à $0,69 \mathrm{~mm}$. L'œsophage est court et large. Les caeca intestinaux sont sinueux, dirigés postérieurement jusqu'à l'extrémité du corps. Le pore Annales de Parasitologie humaine et comparée (Paris), t. 42, 1967, $\mathrm{n}^{\circ} 3$ 


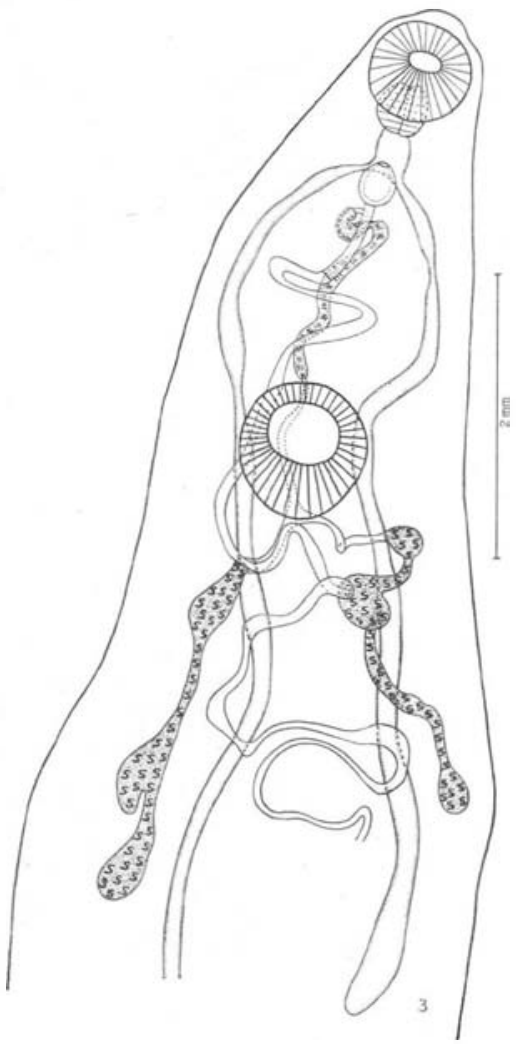

FIG. 3. - Dollfustravassosius moniliovatus gen. n., sp. n. : Paratype $\mathrm{n}^{\circ} 30.082 a$, incomplet, montrant tout l'appareil génital masculin

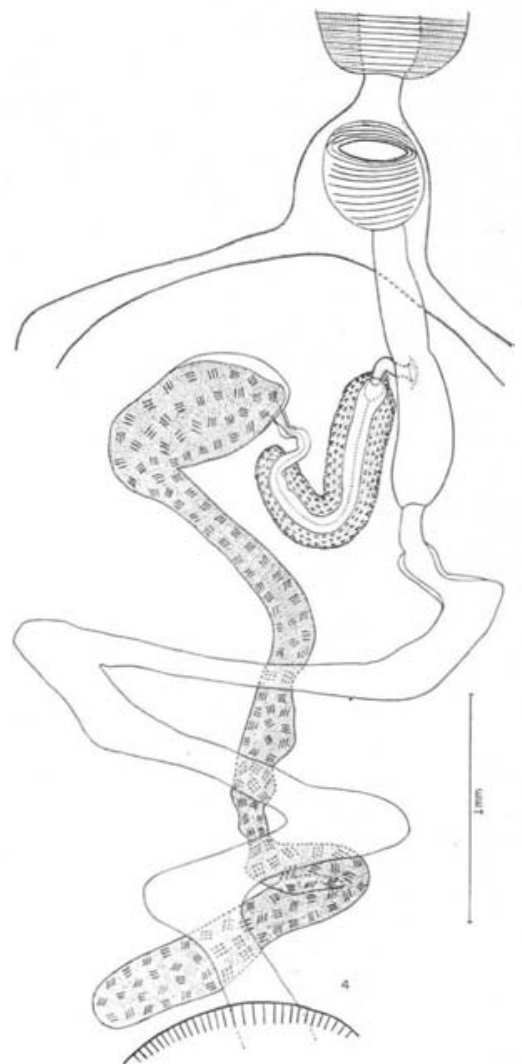

FIG. 4. - Dollfustravassosius moniliovatus gen. n., sp. n. : L'atrium génital, vagine et partie distale de l'appareil génital masculin du paratype $\mathrm{n}^{\circ} 30.082 b$

génital est ventral à l'œsophage. L'atrium génital est tubulaire, plus ou moins cylindrique, long de 0,86 à $1,53 \mathrm{~mm}$ et large de 0,10 à $0,27 \mathrm{~mm}$; sa portion antérieure, longue de 0,28 à $0,35 \mathrm{~mm}$ et large de 0,24 à $0,47 \mathrm{~mm}$, est globuleuse et fortement musculeuse, ayant l'aspect d'un bulbe génital. Il n'y a pas de poche du cirre. Un petit cirre est présent; il pénètre dans l'atrium génital à une distance de 0,33 à $0,67 \mathrm{~mm}$ de son extrémité proximale. La région prostatique est relativement courte. La vessie séminale est longue et sinueuse, atteignant le bord antérieur de la ventouse ventrale. Le canal déférent est long et situé dans l'aire acétabulaire. Les canaux efférents sont plus courts. Les deux testicules sont allongés et post-acétabulaires, présentant ou non des dilatations à leurs extrémités et une dilatation globuleuse, saillante, dans le tiers moyen de sa longueur ; situés latéralement, les testicules occupent les aires intercaecale, 

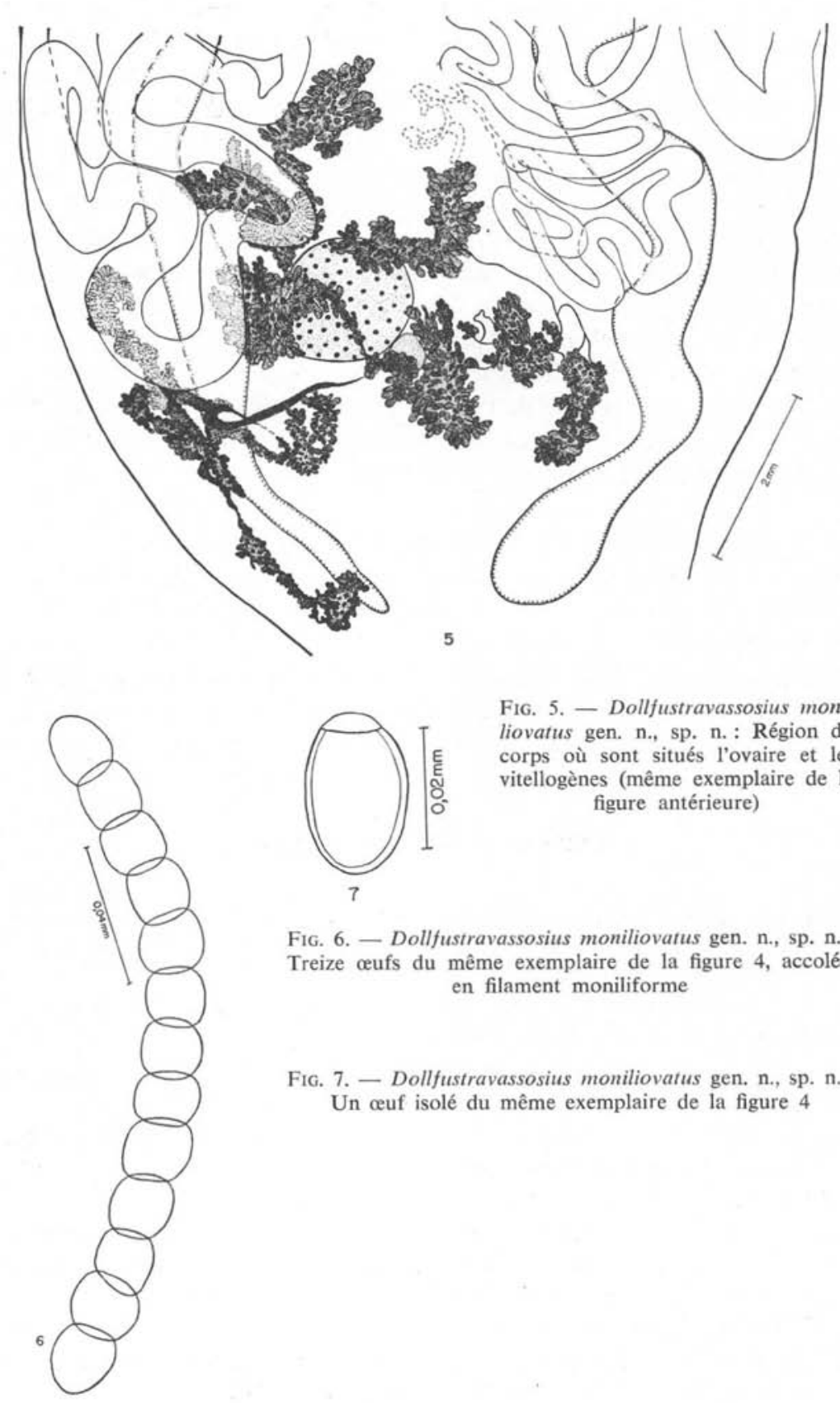

FIG. 5. - Dollfustravassosius moniliovatus gen. n., sp. n.: Région du corps où sont situés l'ovaire et les vitellogènes (même exemplaire de la figure antérieure)

FIG. 6. - Dollfustravassosius moniliovatus gen. n., sp. n. : Treize œufs du même exemplaire de la figure 4, accolés, en filament moniliforme

FIG. 7. - Dollfustravassosius moniliovatus gen. n., sp. n. : Un cuf isolé du même exemplaire de la figure 4 
caecales et extracaecales et mesurent 2,33 à $7,20 \mathrm{~mm}$ de long. L'ovaire est plus ou moins arrondi, intercaecal, rapproché de l'extrémité postérieure du corps, et mesure 0,40 à $1,53 \mathrm{~mm}$ de long sur 0,27 à $1,67 \mathrm{~mm}$ de large. La glande de Mehlis, longue de 0,48 à $0,53 \mathrm{~mm}$ et large de 0,32 à $0,45 \mathrm{~mm}$, est située près de l'ovaire. Le réceptacle séminal féminin (spermathèque) et le canal de Laurer n'ont pas été observés. Les anses utérines sont nombreuses et pré-ovariennes, occupant les aires intercaecale, caecales et extracaecales. Le vagin est long et sinueux, pré-acétabulaire, et s'ouvre à l'extrémité proximale de l'atrium génital. Les œufs, de coloration jaunâtre, sont operculés et se présentent accolés les uns aux autres par leurs extrémités, formant des filaments moniliformes de dimensions variables; ils mesurent 0,015 à $0,024 \mathrm{~mm}$ de long sur 0,012 à $0,020 \mathrm{~mm}$ de large. Les vitellogènes sont constitués par des follicules disposés en grappes, occupant les aires intercaecale, caecales et extracaecales et outrepassant antérieurement et postérieurement la zone de l'ovaire. Le pore excréteur est terminal. La vessie excrétrice est forte et dilatée distalement; elle a la forme d'un $\mathrm{Y}$ et ses branches sont sinueuses et s'anastomosent au niveau du pharynx.

Habitat : Vessie natatoire de Tachysurus grandicassis (Val.).

Provenance: Ile de Marambaia (Océan Atlantique), Etat de Rio de Janeiro, Brésil. TYPE $\mathrm{n}^{\circ} 30.080$ et PARATYPES $\mathrm{n}^{\circ} 30.081$ et 30.082 a-c dans la Collection Helminthologique de l'institut Oswaldo Cruz.

Discussion : Dolfustravassosiinae subfam. n. s'éloigne de la sous-famille Isoparorchiinae (Travassos, 1922) par la forme et par la situation des testicules, par la forme de l'ovaire et par l'aspect des œufs, disposés en filaments moniliformes.

\section{Références bibliographiques}

Dollfus (R. P.), 1959. - Sur un Trématode (genre Isoparorchis), agent pathogène de la maladie de la «tache d'encre » chez des poissons du Vietnam. Bull. Soc. Path. Exot., 52 (6) : 791-803, fig. 1-3.

Skrjabin (K. I.) \& Guschanskaja (L. Kh.), 1955. - Subordre Hemiurata (Markevitsch, 1951) Skrjabin et Guschanskaja, 1954. In Skrjabin, K. I., 1955, Trématodes des animaux et de l'homme, Traité de Trématodologie, 10, 653 pp., 175 fig., Akad. Nauk SSSR ed., Moscou (cf. pp. 339-643, fig. 92-174) (en russe).

Travassos L., 1922. - Contribuições para o conhecimento da fauna helmintológica brasileira. Espécies brasileiras da família Gorgoderidae Looss, 1901. Brasil Medico (36), 1 (2): 17-20.

Travassos L., 1922. - Contribuições para o conhecimento da fauna helmintológica brasileira. XVII. Gorgoderidae brasileiras. Mem. Inst, Oswaldo Cruz, 15 (1) : 220-234, ests,, 26-30, fig. 1-32 (Contributions à l'étude de faune helminthologique du Brésil. XVII. Gorgoderidae brésiliennes : 125-137).

Travassos L., Kohn A. \& Motra C. S., 1963. - Excursão à ilha de Marambaia, Estado do Rio de Janeiro. Atas Soc. Biol. Rio de Janeiro, 7 (3) : 4-9. 
Yamaguti S., 1954. - Systema Helminthum, Part 1, Digenetic trematodes of fishes (1953). II +405 pp., 11 fig., 32 pls, 422 fig., author ed., Tokyo.

Yamaguti S., 1958. - Systema Helminthum, 1, The digenetic trematodes of vertebrates, Part I : XI + 979 pp., Part II : 980-1.232, 1.445-1.575, 106 pl., 1.302 fig., Interscience Publishers, Inc. ed,, New York.

(Instituto Oswaldo Cruz et Conselho Nacional de Pesquisas, Rio de Janeiro, Guanabara, Brasil) 